\title{
Microwave-assisted efficient one-pot synthesis of $N^{2}$-(tetrazol- 5-yl)-6-aryl/heteroaryl-5,6-dihydro-1,3,5-triazine-2,4-diamines
}

\author{
Moustafa Sherief Moustafa ${ }^{1}$, Ramadan Ahmed Mekheimer², \\ Saleh Mohammed Al-Mousawi ${ }^{* 1}$, Mohamed Abd-Elmonem², Hesham El-Zorba ${ }^{3}$, \\ Afaf Mohamed Abdel Hameed ${ }^{2}$, Tahany Mahmoud Mohamed ${ }^{2}$ and Kamal Usef Sadek ${ }^{* 2}$
}

Open Access

\author{
Full Research Paper \\ Address: \\ ${ }^{1}$ Department of Chemistry, Faculty of Science, Kuwait University, P.O. \\ Box 12613, Safat,13060, Kuwait, ${ }^{2}$ Department of Chemistry, Faculty \\ of Science, Minia University, Minia 61519, Egypt and ${ }^{3}$ Department of \\ Pharmacology, Faculty of Veterinary Medicine, Cairo University, Giza \\ 12211, Egypt \\ Email: \\ Saleh Mohammed Al-Mousawi - saleh.almousawi@yahoo.com; \\ Kamal Usef Sadek* - kusadek@yahoo.com \\ * Corresponding author

\section{Keywords:} \\ microwave irradiation; \\ $N^{2}$-(tetrazol-5-yl)-6-aryl/heteroaryl-1,3,5-triazine-2,4-diamines; \\ one-pot synthesis; X-ray crystallography
}

Beilstein J. Org. Chem. 2020, 16, 1706-1712.

doi:10.3762/bjoc. 16.142

Received: 16 April 2020

Accepted: 08 July 2020

Published: 16 July 2020

Associate Editor: I. Baxendale

(C) 2020 Moustafa et al.; licensee Beilstein-Institut. License and terms: see end of document.

\section{Abstract}

An efficient one-pot synthesis of $N^{2}$-(tetrazol-5-yl)-6-aryl/heteroaryl-1,3,5-triazine-2,4-diamine derivatives was developed by reacting 5-amino-1,2,3,4-tetrazole with aromatic aldehydes and cyanamide in pyridine under controlled microwave heating with high yields. X-ray crystallography confirmed the structure of the obtained products.

\section{Introduction}

The family of triazines is of considerable interest in fields related to organic and medicinal chemistry. 2,4-Diaminotriazines are privileged scaffolds exhibiting diverse biological activities such as antibacterial [1], anti-HSV-1 [2], antitumor [3], anti-HIV [4], inhibitor of Trypanosoma brucei [5], angiogenesis inhibitor [6], antiplasmodial antifolates [7], and antimicrobial [8]. Moreover, and in particular $N^{2}, 6$-disubstituted-1,3,5triazine-2,4-diamines possess a wide range of chemotherapeutic activities [8-11].
Tetrazole derivatives are a potent class of heterocyclic compounds with a wide range of biological activities owing to their unique structure. They play an important role not only as a bioisostere of the carboxylic acid group but also as flexible ligands which easily adopt to different binding modes [12,13]. Tetrazole derivatives exhibit a wide spectrum of biological activities as antibacterial [14], anticancer [15], anti-inflammatory [16], antidiabetic [17], antitubercular [18], and analgesic [19] agents. 
It is well established that many medical disorders can be caused as a result of defects at more than one specific biological target such as a receptor or an enzyme. A promising strategy that overcomes the classical one-target, one-molecule approach is the design of stable chemical hybrid molecules which are a combination of two biologically active scaffolds acting at different targets [20-24]. Accordingly, we reasoned that heterocycles incorporating both an $N^{2}$-(tetrazol-5-yl) ring system and a 1,3,5-triazine-2,4-diamine scaffold could be very effective biologically relevant heterocycles.

Little attention has been paid to the synthesis of $N^{2}, 6$-disubstituted-1,3,5-triazine-2,4-diamines which requires a multistep synthesis route. A first approach relied on the nucleophilic substitution of chlorine in cyanuric chloride with Grignard reagents, ammonia or amines $[25,26]$, which suffered from the high reactivity of the Grignard reagents that prevents further functionalization. Moreover, this protocol required temperature control and showed dependence on the amine nucleophile reactivity [27]. Another route involved the reaction of substituted biguanidines with acetic anhydrides, chlorides or carboxylates [11,28-31]. Liu et al. [32] reported a one-pot synthesis of $N^{2}, 6-$ disubstituted-1,3,5-triazine-2,4-diamines in $44-72 \%$ yields that employed the reaction of isothiocyanates with sodium hydrogen cyanamide and amidines in the presence of 1-(3-dimethylaminopropyl)-3-ethylcarbodiimide hydrochloride and heating at $75^{\circ} \mathrm{C}$ for $3 \mathrm{~h}$. Recently, Ma et al. [33] described a one-pot two step procedure for the synthesis of 6-substituted- $N^{2}$-aryl-1,6dihydro-1,3,5-triazine-2,4-diamines via the reaction of aromatic amines, cyanoguanidine, and ketones which afforded the corresponding 1-aryl-1,6-dihydro-6-substituted-1,3,5-triazine-2,4diamines in $21-56 \%$ yields followed by Dimroth rearrangement utilizing sodium hydroxide $(50 \%)$ in aqueous ethanol (Scheme 1).

Pitts et al., yield $70-90 \%$ [25]

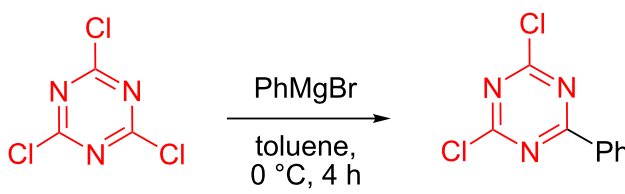<smiles>COc1cc(N)ccc1-c1cnco1</smiles>

THF, rt, $24 \mathrm{~h}$<smiles>COc1cc(Nc2nc(Cl)nc(-c3ccccc3)n2)ccc1-c1cnco1</smiles><smiles>[R]N([R])c1nc(Nc2ccc(-c3cnco3)c(OC)c2)nc(-c2ccccc2)n1</smiles>

Pitts et al., yield 15-35\% [25]

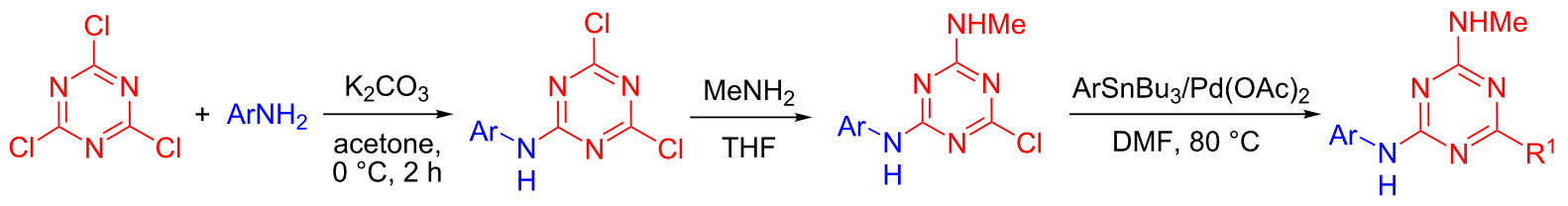

Liu et al., yield $44-72 \%$ [32]

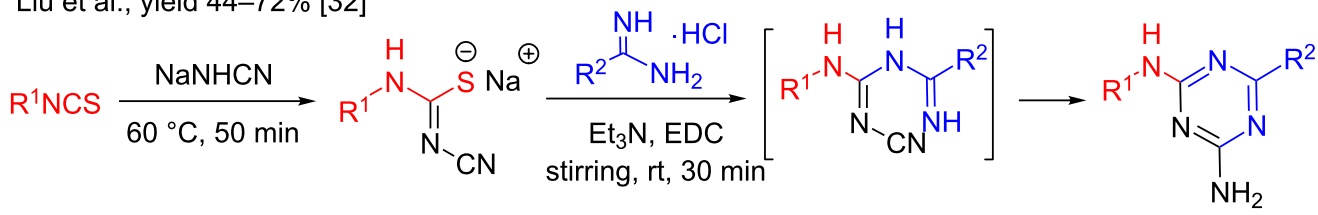

Ma et al., yield $21-56 \%$ [33]<smiles>Nc1c[R1]ccc1</smiles>

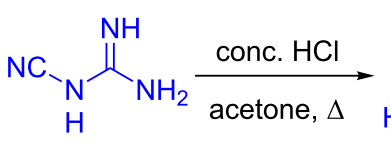<smiles>CC1(Cl)N=C(N)N=C(N)N1c1ccc(F)cc1</smiles>

$\mathrm{NaOH}$ $50 \%$ aq $\mathrm{EtOH}, \Delta$<smiles>[R]c1ccc(NC2=NC(N)=NC(C)(C)N2)cc1</smiles> 
Although, these methods have specific merits, they sometimes suffer from drawbacks such as extended reaction temperatures, lengthy procedures, low yields and atom economy, which consume excess reagents. Extensive efforts have been devoted to adopting green methodologies in synthetic heterocyclic chemistry. The utilization of microwave heating as an energy source has several advantages including operational simplicity, high reaction yields, enhanced rates, and increased energy efficiency [34-40].

In continuation of our efforts in performing green methodologies in the synthesis of biologically relevant heterocycles from simple starting materials [41-44], we developed an efficient synthesis of $N^{2}$-(tetrazol-5-yl)-6-aryl/heteroaryl-1,3,5-triazine2,4-diamines through the one-pot reaction of cyanamide $\mathbf{1}$, aromatic aldehydes $\mathbf{2}$, and 5 -aminotetrazole $(\mathbf{3})$ in pyridine under controlled microwave heating (Scheme 2).

\section{Results and Discussion}

With the initial aim of optimizing the reaction conditions, we began our study by reacting equimolar amounts of cyanamide $\mathbf{1}$, aromatic aldehydes $\mathbf{2}$, and 5-aminotetrazole (3) in pyridine and the reaction was promoted by microwave heating at $120^{\circ} \mathrm{C}$ over $12 \mathrm{~min}$. After cooling the reaction mixture to room temperature and work-up, a solid product was obtained in low yield (40\%) and confirmed to be 6-(4-chlorophenyl)- $N^{2}$-(1H-tetrazol-5-yl)5,6-dihydro-1,3,5-triazine-2,4-diamine (4a) based on analytical and spectral data. The mass spectrum of the reaction product showed a molecular ion peak at $m / z=290.1[\mathrm{M}-1]^{+}$. The ${ }^{1} \mathrm{H}$ NMR revealed four singlet signals at $\delta=11.22,10.81,8.72$, and $6.17 \mathrm{ppm}$ each integrated for one proton which were assigned to the triazine- $\mathrm{NH}, \mathrm{NH}$ at $N^{2}$-(tetrazole-5-yl), tetrazole $\mathrm{NH}$, and triazine $\mathrm{CH}-2$ protons in addition to two broad singlet signals at $\delta=8.59$ and $7.32 \mathrm{ppm}$ for $\mathrm{NH}_{2}$ function as well as signals for the aromatic protons. The ${ }^{13} \mathrm{C}$ NMR spectrum was in support of the proposed structure. Based on the established product, we revealed that two molecules of cyanamide 1 participated in the reaction course and the yield was increased to $92 \%$ when the molar ratio of the reactants $\mathbf{1}, \mathbf{2}$, and $\mathbf{3}$ was set at $2: 1: 1$. We next surveyed a structurally diverse group of aromatic aldehydes $\mathbf{2}$ with cyanamide $\mathbf{1}$ and 5-aminotetrazole (3) under the same experimental conditions and the results are summa- rized in (Table 1 and Scheme 2). Irrespective of the aryl group either electron-donating or electron-withdrawing, the reaction proceeded smoothly and gave a variety of 1,3,5-triazine-2,4-diamines 4 in high yields.

Table 1: Microwave three-component synthesis of triazines $\mathbf{4 a - j}$.

\begin{tabular}{llll} 
Entry & Ar & Product & Yield (\%) \\
\hline 1 & $4-\mathrm{ClC}_{6} \mathrm{H}_{4}$ & $\mathbf{4 a}$ & 92 \\
2 & $4-\mathrm{OMeC}_{6} \mathrm{H}_{4}$ & $\mathbf{4 b}$ & 89 \\
3 & $\mathrm{C}_{6} \mathrm{H}_{5}$ & $\mathbf{4 c}$ & 93 \\
4 & benzo[d]dioxol & $\mathbf{4 d}$ & 88 \\
5 & $2-\mathrm{OMeC}_{6} \mathrm{H}_{4}$ & $\mathbf{4 e}$ & 88 \\
6 & $4-\mathrm{MeC}_{6} \mathrm{H}_{4}$ & $\mathbf{4 f}$ & 87 \\
7 & $2-\mathrm{ClC}_{6} \mathrm{H}_{4}$ & $\mathbf{4 g}$ & 92 \\
8 & $3-\mathrm{NO}_{2} \mathrm{C}_{6} \mathrm{H}_{4}$ & $\mathbf{4 h}$ & 93 \\
9 & $2-$ furyl $_{10}$ & $\mathbf{4 i}$ & 91 \\
& $4-\mathrm{NMe}_{2} \mathrm{C}_{6} \mathrm{H}_{4}$ & $\mathbf{4 j}$ & 88 \\
& & &
\end{tabular}

The effect of the solvent was also examined. Other solvents were screened under the same experimental conditions and the results revealed that performing the reaction in dioxane, $\mathrm{CH}_{3} \mathrm{CN}$, THF, or catalyst-free ethanol resulted in no product formation. However, the same products were obtained with lower yields $(\approx 60 \%)$ when performing the reaction under conventional heating utilizing pyridine as the solvent for 3 hours. These results demonstrated the advantage of microwave heating as an efficient energy source.

The structure proposed for the reaction products was established on the bases of analytical and spectral data (MS, ${ }^{1} \mathrm{H}$ NMR, ${ }^{13} \mathrm{C}$ NMR, and elemental analyses). Moreover, the structure of $4 \mathbf{i}$ was unequivocally supported by single-crystal X-ray diffraction (Figure 1 and Table 2). A plausible mechanism for the formation of products 4 is postulated in Scheme 3.

The dimerization of cyanamide $\mathbf{1}$ in basic medium to cyanoguanidine 5 and subsequent reaction with 5 -aminotetrazole (3) yielded tetrazolylbiguanidine 6 which undergoes a con-

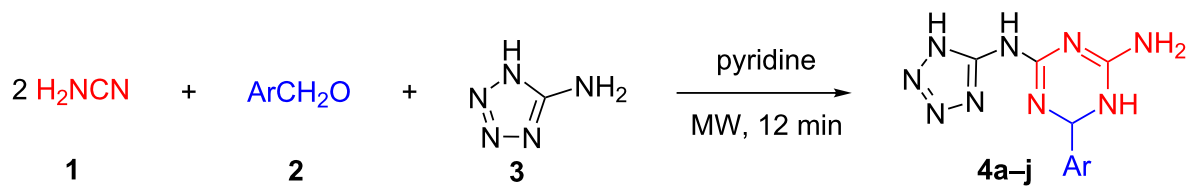




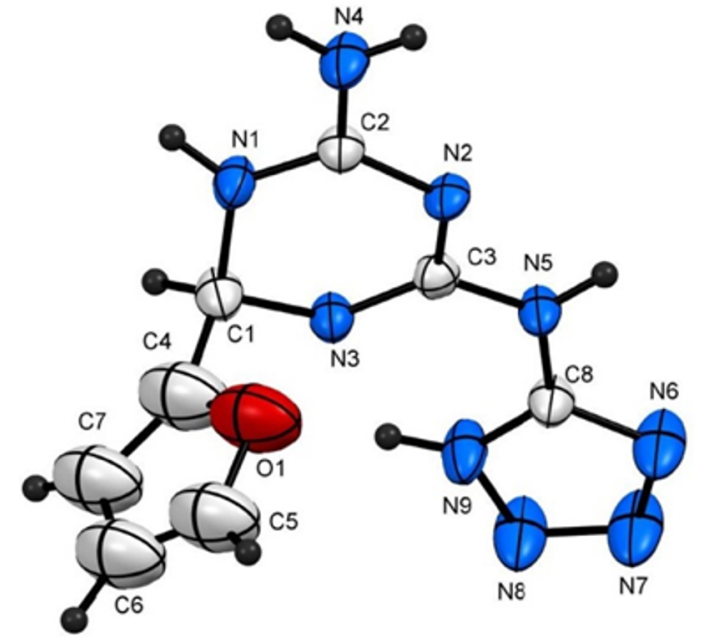

Figure 1: ORTEP diagram of compound $\mathbf{4 i}$.

densation reaction with aromatic aldehydes 2 to afford 7. The nucleophilic attack of the secondary amine in $\mathbf{7}$ to the arylidene carbon gives rise to the formation of 6-aryl-1-( $1 H$-tetrazol-5yl)-1,2-dihydro-1,3,5-triazine-2,4-diamine intermediate 8 (route a) or the nucleophilic attack of the primary amine in $\mathbf{7}$ to the
Table 2: Selected bond lengths and bond angles for compound $\mathbf{4 i}$.

\begin{tabular}{llll}
$\begin{array}{l}\text { atom } \\
\text { numbers }\end{array}$ & $\begin{array}{l}\text { bond lengths } \\
\text { parameter }\left(\mathrm{A}^{\circ}\right)\end{array}$ & $\begin{array}{l}\text { atom } \\
\text { numbers }\end{array}$ & $\begin{array}{l}\text { geometric } \\
\text { parameter }\end{array}$ \\
\hline & & & \\
O1-C5 & $1.360(9)$ & C1-N1-C2 & $121.6(4)$ \\
C4-C7 & $1.326(11)$ & C2-N2-C3 & $115.0(4)$ \\
C1-C4 & $1.465(9)$ & N1-C2-N4 & $118.2(4)$ \\
N6-N7 & $1.336(7)$ & C1-C4-C7 & $136.2(8)$ \\
N5-N3 & $1.339(6)$ & C4-O1-C5 & $109.8(6)$ \\
N1-C1 & $1.453(6)$ & N6-N7-N8 & $109.9(5)$ \\
C1-C4 & $1.465(12)$ & N8-N9-C8 & $104.4(4)$ \\
N3-C1 & $1.459(6)$ & N2-C3-N5 & $116.1(4)$ \\
& & O1-C4-C1 & $116.6(6)$ \\
& & O1-C5-C6 & $106.2(7)$
\end{tabular}

same imine carbon produces the corresponding $N^{2}$-(tetrazole-5yl)-6-aryl-1,3,5-triazine-2,4-diamines 4 (route b). Alternatively, intermediate $\mathbf{8}$ could be obtained by a condensation of 5-aminotetrazole (3) with aromatic aldehydes $\mathbf{2}$ followed by the addition of cyanoguanidine $\mathbf{5}$ to the formed Schiff's base

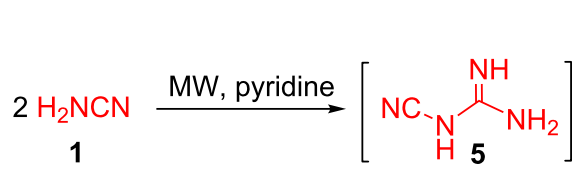<smiles>Nc1nnn[nH]1</smiles><smiles>N=C(N)NC(=N)Nc1nnn[nH]1</smiles>

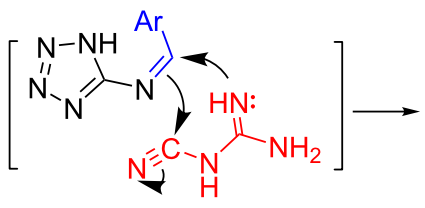<smiles>[B]C1N=C(N)N=C(Br)N1c1nnn[nH]1</smiles>

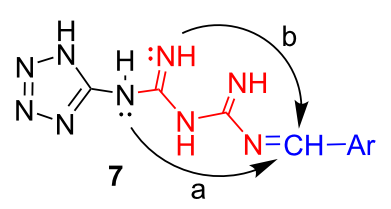<smiles>C[13CH2][13CH3]</smiles>

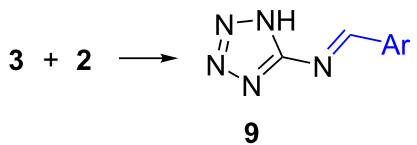<smiles>CCC(C)O</smiles><smiles>C[PbH]</smiles><smiles>NC1=NC(Nc2nnn[nH]2)=NC([Al])N1</smiles>

Scheme 3: Plausible different routes to account for the formation of products 4 . 
(route c). Product 4 was the sole isolable product as under reflux in pyridine as base, compound $\mathbf{8}$ well undergoes a Dimroth rearrangement forming the more thermodynamically stable product 4 [33]. We established route $\mathrm{c}$, as the formation of the Schiff base is more favorable due to the high nucleophilicity of the exocyclic amino function attached to the electronrich tetrazole ring [45]. In support of this assumption we stopped the reaction after 4 minutes of heating under microwave irradiation and inspected the prior formation of 9 via comparison with an authentic sample synthesized by a conventional method.

\section{Conclusion}

The synthesis of biologically relevant 6-aryl/heteroaryl- $N^{2}-(5 H-$ tetrazole-5-yl)-5,6-dihydro-1,3,5-triazine-2,4-diamines was achieved under controlled microwave heating via a simple onepot, three-component reaction of cyanamide $\mathbf{1}$, aldehydes $\mathbf{2}$, and 5-amino-1,2,3,4-tetrazole (3) in excellent yields. The process proved to be an efficient synthetic route displaying high atom economy, short reaction times, and a simple work-up procedure. This protocol appeared to be general with a diversity of amines and aldehydes.

\section{Experimental}

All chemicals were purchased from Aldrich or Merck Companies. The ${ }^{1} \mathrm{H}$ NMR $(600 \mathrm{MHz})$ and ${ }^{13} \mathrm{C}$ NMR $(150 \mathrm{MHz})$ were run in a Bruker DPX instrument ( $\delta \mathrm{ppm})$. Mass spectra were measured by using VG Autospec Q MS 30 and MS 9 (AEI) spectrometer, with EI $(70 \mathrm{eV})$ mode. Melting points were recorded in a Gallenkamp melting point apparatus and are uncorrected. X-ray crystallographic structure determinations were performed by using Rigaku Rapid II and Bruker X8 Prospector single crystal X-ray diffractometers. The X-ray crystal structure data can be obtained free of charge from the Cambridge Crystallographic Data Centre via http://www.ccdc.cam.ac.uk CCDC1961565 for compound 4i. All reactions were monitored by TLC with 1:1 ethyl acetate/petroleum ether as eluent and were carried out until starting materials were completely consumed. After 7 min microwave irradiation was stopped and the reaction mixture was analyzed by TLC; after further irradiation of $5 \mathrm{~min}$ the reaction was complete (total reaction time $12 \mathrm{~min})$.

\section{General procedure for the synthesis of $N^{2}, 6$ - disubstituted dihydro-1,3,5-triazine-2,4- diamine derivatives}

A solution of $\mathbf{1}(2 \mathrm{mmol}), \mathbf{2}(1 \mathrm{mmol})$, and $\mathbf{3}(1 \mathrm{mmol})$ in pyridine $(10 \mathrm{~mL})$ was heated under reflux in a Milestone Microwave Labstation at $120{ }^{\circ} \mathrm{C}$ for $12 \mathrm{~min}$. The solvent was removed under reduced pressure and the solid product was isolated by filtration and recrystallized from DMF.
6-(4-Chlorophenyl)- $\mathrm{N}^{\mathbf{2}}$-(5H-tetrazol-5-yl)-5,6-dihydro-1,3,5triazine-2,4-diamine (4a). Colorless crystals; mp 320-322 ${ }^{\circ} \mathrm{C}$; yield $0.268 \mathrm{~g}, 92 \% ; R_{\mathrm{f}} 0.55$ (1:1 ethyl acetate/petroleum ether); ${ }^{1} \mathrm{H}$ NMR (600 MHz, DMSO- $\left.d_{6}\right) \delta 6.17(\mathrm{~s}, 1 \mathrm{H}), 7.32(\mathrm{~s}, 1 \mathrm{H})$, $7.46(\mathrm{~d}, J=8.4 \mathrm{~Hz}, 2 \mathrm{H}), 7.53(\mathrm{~d}, J=9.2 \mathrm{~Hz}, 2 \mathrm{H}), 8.58(\mathrm{~s}, 1 \mathrm{H})$, 8.73 (brs, $1 \mathrm{H}), 10.82(\mathrm{~s}, 1 \mathrm{H}), 11.22(\mathrm{~s}, 1 \mathrm{H}) ;{ }^{13} \mathrm{C} \mathrm{NMR}$ $\left(150 \mathrm{MHz}, \mathrm{DMSO}-d_{6}\right) \delta 61.52,127.84,128.94,133.70,139.75$, 154.45, 157.61, 158.0; anal. calcd for $\mathrm{C}_{10} \mathrm{H}_{10} \mathrm{ClN}_{9}$ : C, 41.17; $\mathrm{H}$, 3.46; Cl, 12.15; N, 43.22; found: C, 41.22; H, 3.42; Cl, 12.11; $\mathrm{N}, 43.24$; $\operatorname{EIMS}(\mathrm{m} / \mathrm{z}): 290.1[\mathrm{M}-1]^{+}$.

6-(4-Methoxyphenyl)- $N^{2}$-(5H-tetrazol-5-yl)-5,6-dihydro1,3,5-triazine-2,4-diamine (4b). Colorless crystals; mp 306-308 ${ }^{\circ} \mathrm{C}$; yield $0.255 \mathrm{~g}, 89 \%$; ${ }^{1} \mathrm{H}$ NMR (600 MHz, DMSO$\left.d_{6}\right) \delta 3.74(\mathrm{~s}, 3 \mathrm{H}), 6.07(\mathrm{~s}, 1 \mathrm{H}), 7.01(\mathrm{~d}, J=8.4, \mathrm{~Hz}, 2 \mathrm{H}), 7.38$ (br s, 1H), $7.39(\mathrm{~d}, J=9 \mathrm{~Hz}, 2 \mathrm{H}), 8.29(\mathrm{~s}, 1 \mathrm{H}), 8.60(\mathrm{~s}, 1 \mathrm{H})$, $10.69(\mathrm{~s}, 1 \mathrm{H}), 11.15(\mathrm{~s}, 1 \mathrm{H}) ;{ }^{13} \mathrm{C}$ NMR $\left(150 \mathrm{MHz}, \mathrm{DMSO}-d_{6}\right) \delta$ 55.26, 62.01, 114.29, 127.51, 132.45, 154.57, 157.79, 158.14, 159.92; anal. calcd for $\mathrm{C}_{11} \mathrm{H}_{13} \mathrm{~N}_{9} \mathrm{O}: \mathrm{C}, 45.99 ; \mathrm{H}, 4.56$; N, 43.88; found: C, 45.89; H, 4.52; N, 43.90; $\operatorname{EIMS~}(\mathrm{m} / \mathrm{z}): 286.1$ $[\mathrm{M}-1]^{+}$.

6-Phenyl- $N^{2}$-(5H-tetrazol-5-yl)-5,6-dihydro-1,3,5-triazine2,4-diamine (4c). Colorless crystals; mp 317-319 ${ }^{\circ} \mathrm{C}$; yield $0.277 \mathrm{~g}, 93 \%$; ${ }^{1} \mathrm{H}$ NMR (600 MHz, DMSO- $\left.d_{6}\right) \delta 6.15(\mathrm{~s}, 1 \mathrm{H})$, $7.22(\mathrm{~s}, 1 \mathrm{H}), 7.40-7.46(\mathrm{~m}, 5 \mathrm{H}), 8.50(\mathrm{br} \mathrm{s}, 1 \mathrm{H}), 8.71(\mathrm{~s}, 1 \mathrm{H})$, 10.77 (s, 1H), $11.20(\mathrm{~s}, 1 \mathrm{H}) ;{ }^{13} \mathrm{C}$ NMR $\left(150 \mathrm{MHz}, \mathrm{DMSO}-d_{6}\right) \delta$ $62.2,125.9,128.9,129.1,154.5,157.7,158.0$; anal. calcd for $\mathrm{C}_{10} \mathrm{H}_{11} \mathrm{~N}_{9}$ : C, 46.69; $\mathrm{H}, 4.31$; N, 49.0; found: $\mathrm{C}, 46.76$; $\mathrm{H}, 4.41$; $\mathrm{N}, 48.85$; $\operatorname{EIMS}(\mathrm{m} / \mathrm{z}): 256.1[\mathrm{M}-1]^{+}$.

6-(Benzo $[d][1,3] \operatorname{dioxol-5}-y \mathrm{l})-N^{2}-(5 H$-tetrazol-5-yl)-5,6dihydro-1,3,5-triazine-2,4-diamine (4d). Colorless crystals; mp 304-306 ${ }^{\circ} \mathrm{C}$; yield $0.265 \mathrm{~g}, 88 \%$; ${ }^{1} \mathrm{H}$ NMR $(600 \mathrm{MHz}$, DMSO- $\left.d_{6}\right) \delta, 6.02(\mathrm{~s}, 1 \mathrm{H}), 6.04(\mathrm{~d}, 2 \mathrm{H}), 6.90-7.12(\mathrm{~m}, 4 \mathrm{H})$, 8.02 (brs, 1H), $8.55(\mathrm{~s}, 1 \mathrm{H}), 10.68(\mathrm{~s}, 1 \mathrm{H}), 11.10(\mathrm{~s}, 1 \mathrm{H})$; ${ }^{13} \mathrm{C}$ NMR $\left(150 \mathrm{MHz}, \mathrm{DMSO}-d_{6}\right) \delta 56.0,62.0,101.4,106.3$, $108.29,119.63,134.38,147.76,147.93,154.49,157.72$, 158.15; anal. calcd for $\mathrm{C}_{11} \mathrm{H}_{11} \mathrm{~N}_{9} \mathrm{O}_{2}: \mathrm{C}, 43.85 ; \mathrm{H}, 3.68 ; \mathrm{N}$, 41.84; found: C, 43.76; H, 3.59, N, 41.79.

6-(2-Methoxyphenyl)- $N^{2}$-(5H-tetrazol-5-yl)-5,6-dihydro1,3,5-triazine-2,4-diamine (4e). Colorless crystals; $\mathrm{mp}$ 310-312 ${ }^{\circ} \mathrm{C}$; yield $0.247 \mathrm{~g}, 88 \%$; ${ }^{1} \mathrm{H}$ NMR $(600 \mathrm{MHz}$, DMSO$\left.d_{6}\right) \delta 3.89(\mathrm{~s}, 3 \mathrm{H}), 6.27(\mathrm{~s}, 1 \mathrm{H}), 7.02(\mathrm{t}, J=8.4 \mathrm{~Hz}, 1 \mathrm{H}), 7.12(\mathrm{~d}$, $J=8.4 \mathrm{~Hz}, 1 \mathrm{H}), 7.20,7.21(\mathrm{dd}, J=8.4,1.8 \mathrm{~Hz}, 1 \mathrm{H}), 7.38-7.41$ (m, 1H), 8.04 (brs, 1H), $8.36(\mathrm{~s}, 1 \mathrm{H}), 10.87$ (s, 1H), 11.07 (s, $1 \mathrm{H}) ;{ }^{13} \mathrm{C}$ NMR $\left(150 \mathrm{MHz}, \mathrm{DMSO}-d_{6}\right) \delta 55.85,58.74,111.65$, 120.42, 125.28, 127.77, 130.50, 154.61, 156.3, 157.97, 158.2; anal. calcd for $\mathrm{C}_{11} \mathrm{H}_{13} \mathrm{~N}_{9} \mathrm{O}: \mathrm{C}, 45.99 ; \mathrm{H}, 4.56 ; \mathrm{N}, 43.88$; found: C, 46.10; H, 4.69; N, 43.81; EIMS (m/z): $286.1[\mathrm{M}-1]^{+}$. 
6-(4-Methylphenyl)- $N^{\mathbf{2}}$-(5H-tetrazol-5-yl)-5,6-dihydro-1,3,5triazine-2,4-diamine (4f). Colorless crystals; mp $314-316{ }^{\circ} \mathrm{C}$; yield $0.235 \mathrm{~g}, 87 \% ;{ }^{1} \mathrm{H}$ NMR (600 MHz, DMSO- $\left.d_{6}\right) \delta 2.50$ (s, $3 \mathrm{H}), 6.09$ (s, 1H), 7.19 (s, 1H), 7.26 (d, J = 8.4 Hz, 2H), 7.34 (d, $J=8.4 \mathrm{~Hz}, 2 \mathrm{H}), 8.47$ (brs, 1H), 8.66 (s, 1H), 10.7 (s, 1H), 11.18 $(\mathrm{s}, 1 \mathrm{H}) ;{ }^{13} \mathrm{C}$ NMR $\left(150 \mathrm{MHz}, \mathrm{DMSO}-d_{6}\right) \delta 20.73,62.08$, 125.90, 129.41, 137.64, 138.69, 154.53, 157.76, 158.07; anal. calcd for $\mathrm{C}_{11} \mathrm{H}_{13} \mathrm{~N}_{9}$ : C, 48.70; H, 4.83; N, 46.47; found: C, $48.75 ; \mathrm{H}, 4.70 ; \mathrm{N}, 46.56$.

6-(2-Chlorophenyl)- $N^{\mathbf{2}}$-(5H-tetrazol-5-yl)-5,6-dihydro-1,3,5triazine-2,4-diamine (4g). Colorless crystals; mp $324-326{ }^{\circ} \mathrm{C}$; yield $0.267 \mathrm{~g}, 92 \% ;{ }^{1} \mathrm{H}$ NMR (600 MHz, DMSO- $\left.d_{6}\right) \delta 6.44$ (s, $1 \mathrm{H}), 7.22(\mathrm{~s}, 1 \mathrm{H}), 7.42-7.44(\mathrm{~m}, 1 \mathrm{H}), 7.45-7.48(\mathrm{~m}, 2 \mathrm{H})$, $7.56-7.59(\mathrm{~m}, 1 \mathrm{H}), 8.55$ (brs, 1H), $8.65(\mathrm{~s}, 1 \mathrm{H}), 10.94(\mathrm{~s}, 1 \mathrm{H})$, $11.29(\mathrm{~s}, 1 \mathrm{H}) ;{ }^{13} \mathrm{C}$ NMR $\left(150 \mathrm{MHz}, \mathrm{DMSO}-d_{6}\right) \delta 60.55$, 127.39, 127.98, 130.30, 131.05, 131.42, 137.07, 154.52, 157.71, 158.04, 162.31; anal. calcd for $\mathrm{C}_{10} \mathrm{H}_{10} \mathrm{ClN}_{9}$ : C, 41.17; $\mathrm{H}, 3.46$; $\mathrm{Cl}, 12.15 ; \mathrm{N}, 43.22$; found: $\mathrm{C}, 41.30 ; \mathrm{H}, 3.34 ; \mathrm{Cl}, 12.30 ; \mathrm{N}$, 43.38 .

6-(3-Nitrophenyl)- $N^{2}$-(5H-tetrazol-5-yl)-5,6-dihydro-1,3,5triazine-2,4-diamine (4h). Colorless crystals; mp $298-300{ }^{\circ} \mathrm{C}$; yield $0.281 \mathrm{~g}, 93 \%$; ${ }^{1} \mathrm{H}$ NMR (600 MHz, DMSO- $\left.d_{6}\right) \delta 6.35$ (s, $1 \mathrm{H}), 7.52(\mathrm{~s}, 1 \mathrm{H}), 7.76(\mathrm{t}, J=7.2 \mathrm{~Hz}, 1 \mathrm{H}), 7.88(\mathrm{~s}, 1 \mathrm{H}), 8.26(\mathrm{~d}$, $J=7.8 \mathrm{~Hz}, 1 \mathrm{H}), 8.34$ (s, 1H), 8.88 (brs, 2H), 10.95 (s, 1H), $11.26(\mathrm{~s}, 1 \mathrm{H}) ;{ }^{13} \mathrm{C} \mathrm{NMR}\left(150 \mathrm{MHz}, \mathrm{DMSO}-d_{6}\right) \delta 61.28$, 120.92, 123.91, 130.73, 132.27, 143.13, 147.95, 154.42, 157.62, 157.94; anal. calcd for $\mathrm{C}_{10} \mathrm{H}_{10} \mathrm{~N}_{10} \mathrm{O}_{2}$ : C, 39.74; $\mathrm{H}, 3.33 ; \mathrm{N}$, 46.34; found: C, 39.68; H, 3.47; N, 46.52.

6-(Furan-2-yl)- $\mathrm{N}^{\mathbf{2}}$-(5H-tetrazol-5-yl)-5,6-dihydro-1,3,5triazine-2,4-diamine (4i). Colorless crystals; mp $208-210{ }^{\circ} \mathrm{C}$; yield $0.224 \mathrm{~g}, 91 \%$; ${ }^{1} \mathrm{H}$ NMR (600 MHz, DMSO- $\left.d_{6}\right) \delta 6.24$ (s, $1 \mathrm{H}), 6.46$ (d, $J=16.8 \mathrm{~Hz}, 2 \mathrm{H}), 7.29$ (brs, $1 \mathrm{H}), 7.70$ (s, 1H), 8.52 (brs, 1H), $8.69(\mathrm{~s}, 1 \mathrm{H}), 10.76(\mathrm{~s}, 1 \mathrm{H}), 11.21(\mathrm{~s}, 1 \mathrm{H}) ;{ }^{13} \mathrm{C} \mathrm{NMR}$ $\left(150 \mathrm{MHz}, \mathrm{DMSO}-d_{6}\right) \delta 56.48,107.88,110.61,143.88,152.35$, 154.52, 157.68, 157.97; anal. calcd for $\mathrm{C}_{8} \mathrm{H}_{9} \mathrm{~N}_{9} \mathrm{O}$ : C, 38.87; $\mathrm{H}$, 3.67; N, 50.99; found: C, 38.68; H, 3.76; N, 51.06; EIMS ( $\mathrm{m} / \mathrm{z})$ : $246.1[\mathrm{M}-1]^{+}$.

6-(4- $N, N$-Dimethylaminophenyl)- $N^{2}$-(5H-tetrazol-5-yl)-5,6dihydro-1,3,5-triazine-2,4-diamine $(\mathbf{4 j})$. Colorless crystals; mp 278-280 ${ }^{\circ} \mathrm{C}$; yield $0.264 \mathrm{~g}, 88 \%$; ${ }^{1} \mathrm{H}$ NMR $(600 \mathrm{MHz}$, DMSO- $\left.d_{6}\right) \delta 2.91(\mathrm{~s}, 6 \mathrm{H}), 5.96(\mathrm{~s}, 1 \mathrm{H}), 6.59(\mathrm{~d}, J=8.4 \mathrm{~Hz}$, 2H), $7.01(\mathrm{~s}, 1 \mathrm{H}), 7.20(\mathrm{~d}, J=8.4 \mathrm{~Hz}, 2 \mathrm{H}), 7.78$ (brs, $1 \mathrm{H}), 8.46$ (s, 1H), 10.59 (s, 1H), $11.03(\mathrm{~s}, 1 \mathrm{H}) ;{ }^{13} \mathrm{C} \mathrm{NMR}(150 \mathrm{MHz}$, DMSO- $d_{6}$ ) $\delta 39.97,40.03,62.36,111.42,112.15,127.01$, $127.17,128.32,151.04,154.64,157.90,158.24$; anal. calcd for $\mathrm{C}_{12} \mathrm{H}_{16} \mathrm{~N}_{10}$ : C, 47.99; $\mathrm{H}, 5.37$; N, 46.64; found: $\mathrm{C}, 48.10 ; \mathrm{H}$, $5.43 ; \mathrm{N}, 46.52$.

\section{Supporting Information}

\section{Supporting Information File 1}

NMR and mass spectra.

[https://www.beilstein-journals.org/bjoc/content/

supplementary/1860-5397-16-142-S1.pdf]

\section{Funding}

K. U. Sadek is grateful to the Alexander von Humboldt Foundation for donation of a Milestone START Microwave Labstation. Saleh Al-Mousawi and Moustafa Sherief Moustafa are grateful to the Kuwait Foundation for the Advancement of Science, project number PR1714SC02 for supporting this work. Analytical facilities provided by Kuwait University GFS projects No. GS 01/03 and GS 03/08 are greatly appreciated.

\section{ORCID ${ }^{\circledR}$ iDs}

Moustafa Sherief Moustafa - https://orcid.org/0000-0002-3694-5845 Ramadan Ahmed Mekheimer - https://orcid.org/0000-0003-4685-0927 Mohamed Abd-Elmonem - https://orcid.org/0000-0002-4808-1650 Kamal Usef Sadek - https://orcid.org/0000-0003-4342-5394

\section{Preprint}

A non-peer-reviewed version of this article has been previously published as a preprint doi:10.3762/bxiv.2020.51.v1

\section{References}

1. Nishigaki, S.; Yoneda, F.; Matsumoto, H.; Morinaga, K. J. Med. Chem. 1969, 12, 39-42. doi:10.1021/jm00301a010

2. Goda, F.; Tantawy, A.; Abouzeid, L.; Badr, S.; Selim, K. Saudi Pharm. J. 2008, 16, 103-111.

3. Sączewski, F.; Bułakowska, A.; Bednarski, P.; Grunert, R. Eur. J. Med. Chem. 2006, 41, 219-225. doi:10.1016/j.ejmech.2005.10.013

4. Chen, X.; Zhan, P.; Liu, X.; Cheng, Z.; Meng, C.; Shao, S.; Pannecouque, C.; Clercq, E. D.; Liu, X. Bioorg. Med. Chem. 2012, 20 , 3856-3864. doi:10.1016/j.bmc.2012.04.030

5. Landi, G.; Linciano, P.; Borsari, C.; Bertolacini, C. P.; Moraes, C. B.; Cordeiro-da-Silva, A.; Gul, S.; Witt, G.; Kuzikov, M.; Costi, M. P.; Pozzi, C.; Mangani, S. ACS Infect. Dis. 2019, 5, 1105-1114. doi:10.1021/acsinfecdis.8b00358

6. Poirier, M.; Awale, M.; Roelli, M. A.; Giuffredi, G. T.; Ruddigkeit, L.; Evensen, L.; Stooss, A.; Calarco, S.; Lorens, J. B.; Charles, R.-P.; Reymond, J.-L. ChemMedChem 2019, 14, 224-236. doi:10.1002/cmdc.201800554

7. Lourens, A. C. U.; Gravestock, D.; van Zyl, R. L.; Hoppe, H. C.; Kolesnikova, N.; Taweechai, S.; Yuthavong, Y.; Kamchonwongpaisan, S.; Rousseau, A. L. Org. Biomol. Chem. 2016, 14, 7899-7911. doi:10.1039/c6ob01350c

8. Ma, X.; Tan, S.-T.; Khoo, C.-L.; Sim, H.-M.; Chan, L.-W.; Chui, W.-K. Bioorg. Med. Chem. Lett. 2011, 21, 5428-5431. doi:10.1016/j.bmcl.2011.06.125 
9. Hanessian, S.; Sgarbi, P. W. M. Bioorg. Med. Chem. Lett. 2000, 10, 433-437. doi:10.1016/s0960-894x(00)00021-4

10. Hajduk, P. J.; Dinges, J.; Schkeryantz, J. M.; Janowick, D.; Kaminski, M.; Tufano, M.; Augeri, D. J.; Petros, A.; Nienaber, V.; Zhong, P.; Hammond, R.; Coen, M.; Beutel, B.; Katz, L.; Fesik, S. W. J. Med. Chem. 1999, 42, 3852-3859. doi:10.1021/jm990293a

11. Kosáry, J.; Kasztreiner, E.; Rablóczky, G.; Kürthy, M. Eur. J. Med. Chem. 1989, 24, 97-99. doi:10.1016/0223-5234(89)90171-2

12. Burger, A. Isosterism and bioisosterism in drug design. In Progress in Drug Research; Jucker, E., Ed.; Birkhäuser: Basel, Switzerland, 2011; Vol. 37, pp 287-371. doi:10.1007/978-3-0348-7139-6_7

13. Ostrovskii, V. A.; Trifonov, R. E.; Popova, E. A. Russ. Chem. Bull. 2012, 61, 768-780. doi:10.1007/s11172-012-0108-4

14. Malik, M. A.; Al-Thabaiti, S. A.; Malik, M. A. Int. J. Mol. Sci. 2012, 13, 10880-10898. doi:10.3390/ijms130910880

15. Muralikrishna, S.; Raveendrareddy, P.; Ravindranath, L. K.; Harikrishna, S.; Pathange, J. Der Pharm. Chem. 2013, 5 (6), 87-93.

16. Ostrovskii, V. A.; Koren, A. O. Heterocycles 2000, 53, 1421-1448. doi:10.3987/rev-00-530

17. Mohite, P. B.; Bhaskar, V. H. Int. J. PharmTech Res. 2011, 3, 1557-1566.

18. Adamec, J.; Waisser, K.; Kuneš, J.; Kaustová, J. Arch. Pharm. (Weinheim, Ger.) 2005, 338, 385-389. doi:10.1002/ardp.200400967

19. Bachar, S. C.; Lahiri, S. C. Pharmazie 2004, 59, 435-438.

20. Decker, M. Design of Hybrid Molecules for Drug Development; Elsevier: New York, NY, USA, 2017.

21. Shaveta; Mishra, S.; Singh, P. Eur. J. Med. Chem. 2016, 124, 500-536. doi:10.1016/j.ejmech.2016.08.039

22. Solomon, V. R.; Hu, C.; Lee, H. Bioorg. Med. Chem. 2009, 17, 7585-7592. doi:10.1016/j.bmc.2009.08.068

23. Kelly, P. M.; Keely, N. O.; Bright, S. A.; Yassin, B.; Ana, G.; Fayne, D.; Zisterer, D. M.; Meegan, M. J. Molecules 2017, 22, 1440. doi:10.3390/molecules22091440

24. Pingaew, R.; Saekee, A.; Mandi, P.; Nantasenamat, C.; Prachayasittikul, S.; Ruchirawat, S.; Prachayasittikul, V. Eur. J. Med. Chem. 2014, 85, 65-76. doi:10.1016/j.ejmech.2014.07.087

25. Pitts, W. J.; Guo, J.; Dhar, T. G. M.; Shen, Z.; Gu, H. H.; Watterson, S. H.; Bednarz, M. S.; Chen, B.-C.; Barrish, J. C.; Bassolino, D.; Cheney, D.; Fleener, C. A.; Rouleau, K. A.; Hollenbaugh, D. L.; Iwanowicz, E. J. Bioorg. Med. Chem. Lett. 2002, 12, 2137-2140. doi:10.1016/s0960-894x(02)00351-7

26. Hirt, R.; Nidecker, H.; Berchtold, R.; Schönholzer, G. Helv. Chim. Acta 1950, 33, 1365-1369. doi:10.1002/hlca.19500330536

27. List, M.; Puchinger, H.; Gabriel, H.; Monkowius, U.; Schwarzinger, C. J. Org. Chem. 2016, 81, 4066-4075. doi:10.1021/acs.joc.6b00355

28. Alkalay, D.; Volk, J.; Bartlett, M. F. J. Pharm. Sci. 1976, 65, 525-529. doi:10.1002/jps.2600650412

29. Yuki, Y.; Sakurai, S.; Kakurai, T.; Noguchi, T. Bull. Chem. Soc. Jpn. 1970, 43, 2130-2134. doi:10.1246/bcsj.43.2130

30. Shapiro, S. L.; Isaacs, E. S.; Parrino, V. A.; Freedman, L. J. Org. Chem. 1961, 26, 68-74. doi:10.1021/jo01060a015

31. Irikura, T.; Abe, Y.; Okamura, K.; Higo, K.; Maeda, A.; Morinaga, F.; Shirai, G.; Hatae, S. J. Med. Chem. 1970, 13, 1081-1089. doi:10.1021/jm00300a015

32. Liu, C.; Lin, J.; Leftheris, K. Tetrahedron Lett. 2007, 48, 435-437. doi:10.1016/j.tetlet.2006.11.069
33. Ma, X.; Poon, T.-Y.; Wong, P. T. H.; Chui, W.-K. Bioorg. Med. Chem. Lett. 2009, 19, 5644-5647. doi:10.1016/j.bmcl.2009.08.052

34. Carpenter, R. D.; Lam, K. S.; Kurth, M. J. J. Org. Chem. 2007, 72, 284-287. doi:10.1021/jo0618066

35. Caddick, S. Tetrahedron 1995, 51, 10403-10432. doi:10.1016/0040-4020(95)00662-r

36. Hameed, A. A.; Ahmed, E. K.; Fattah, A. A. A.; Andrade, C. K. Z.; Sadek, K. U. Res. Chem. Intermed. 2017, 43, 5523-5533. doi:10.1007/s11164-017-2944-1

37. Sadek, K. U.; Mekheimer, R. A.; Mohamed, T. M.; Moustafa, M. S.; Elnagdi, M. H. Beilstein J. Org. Chem. 2012, 8, 18-24. doi:10.3762/bjoc.8.3

38. Hayes, B. L. Microwave Synthesis: Chemistry at the Speed of Light; CEM Publishing: NC, USA, 2002.

39. Kaval, N.; Ermolat'ev, D.; Appukkuttan, P.; Dehaen, W.; Kappe, C. O.; Van der Eycken, E. J. Comb. Chem. 2005, 7, 490-502. doi:10.1021/cc0498377

40. Nüchter, M.; Ondruschka, B.; Bonrath, W.; Gum, A. Green Chem. 2004, 6, 128-141. doi:10.1039/b310502d

41. Abdel Hameed, A. M.; Moustafa, M. S.; Al-Mousawi, S. M.; Awed, R. R.; Sadek, K. U. Green Process. Synth. 2017, 6, 371-375. doi:10.1515/gps-2017-0019

42. Mekheimer, R. A.; Hameed, A. A.; Sadek, K. U. Green Chem. 2008, 10, 592-593. doi:10.1039/b715126h

43. Dyab, A. K. F.; Sadek, K. U. RSC Adv. 2018, 8, 23241-23251. doi:10.1039/c8ra04195d

44. Abdel Hamid, A.; Abd-Elmonem, M.; Hayallah, A. M.; Abo Elsoud, F. A.; Sadek, K. U. ChemistrySelect 2017, 2, 10689-10693. doi:10.1002/slct.201702011

45. Ostrovskii, V. A.; Koldobskii, G. I.; Trifonov, R. E. Tetrazoles. Comprehensive Heterocyclic Chemistry III; Elsevier: Amsterdam, Netherlands, 2008; Vol. 6, pp 257-423. doi:10.1016/b978-008044992-0.00517-4

\section{License and Terms}

This is an Open Access article under the terms of the Creative Commons Attribution License (http://creativecommons.org/licenses/by/4.0). Please note that the reuse, redistribution and reproduction in particular requires that the authors and source are credited.

The license is subject to the Beilstein Journal of Organic Chemistry terms and conditions: (https://www.beilstein-journals.org/bjoc)

The definitive version of this article is the electronic one which can be found at: doi: $10.3762 /$ bjoc. 16.142 